\title{
Magnetic properties of hematite nanoparticles
}

\author{
Bødker, Franz; Hansen, Mikkel Fougt; Bender Koch, Christian; Lefmann, Kim; Mørup, Steen
}

Published in:

Physical Review B Condensed Matter

Link to article, DOI:

10.1103/PhysRevB.61.6826

Publication date:

2000

Document Version

Publisher's PDF, also known as Version of record

Link back to DTU Orbit

Citation (APA):

Bødker, F., Hansen, M. F., Bender Koch, C., Lefmann, K., \& Mørup, S. (2000). Magnetic properties of hematite nanoparticles. Physical Review B Condensed Matter, 61(10), 6826-6838.

https://doi.org/10.1103/PhysRevB.61.6826

\section{General rights}

Copyright and moral rights for the publications made accessible in the public portal are retained by the authors and/or other copyright owners and it is a condition of accessing publications that users recognise and abide by the legal requirements associated with these rights.

- Users may download and print one copy of any publication from the public portal for the purpose of private study or research.

- You may not further distribute the material or use it for any profit-making activity or commercial gain

- You may freely distribute the URL identifying the publication in the public portal

If you believe that this document breaches copyright please contact us providing details, and we will remove access to the work immediately and investigate your claim. 


\title{
Magnetic properties of hematite nanoparticles
}

\author{
Franz Bødker and Mikkel F. Hansen \\ Department of Physics, Building 307, Technical University of Denmark, DK-2800 Lyngby, Denmark
}

Christian Bender Koch

Department of Chemistry, The Royal Veterinary and Agricultural University, Thorvaldsensvej 40, DK-1871 Frederiksberg C, Denmark

Kim Lefmann

Department of Condensed Matter Physics and Chemistry, Ris $\phi$ National Laboratory, DK-4000 Roskilde, Denmark

\section{Steen Mørup}

Department of Physics, Building 307, Technical University of Denmark, DK-2800 Lyngby, Denmark

(Received 28 July 1998; revised manuscript received 11 November 1999)

\begin{abstract}
The magnetic properties of hematite $\left(\alpha-\mathrm{Fe}_{2} \mathrm{O}_{3}\right)$ particles with sizes of about $16 \mathrm{~nm}$ have been studied by use of Mössbauer spectroscopy, magnetization measurements, and neutron diffraction. The nanoparticles are weakly ferromagnetic at temperatures at least down to $5 \mathrm{~K}$ with a spontaneous magnetization that is only slightly higher than that of weakly ferromagnetic bulk hematite. At $T \gtrsim 100 \mathrm{~K}$ the Mössbauer spectra contain a doublet, which is asymmetric due to magnetic relaxation in the presence of an electric field gradient in accordance with the Blume-Tjon model. Simultaneous fitting of series of Mössbauer spectra obtained at temperatures from $5 \mathrm{~K}$ to well above the superparamagnetic blocking temperature allowed the estimation of the pre-exponential factor in Néel's expression for the superparamagnetic relaxation time, $\tau_{0}=(6 \pm 4) \times 10^{-11} \mathrm{~s}$ and the magnetic anisotropy energy barrier, $E_{\mathrm{bm}} / k=590_{-120}^{+150} \mathrm{~K}$. A lower value of the pre-exponential factor, $\tau_{0}=1.8_{-1.3}^{+3.2} \times 10^{-11} \mathrm{~s}$, and a significantly lower anisotropy energy barrier $E_{\mathrm{bm}}^{\mathrm{magn}} / k=305 \pm 20 \mathrm{~K}$ was derived from simultaneous fitting to ac and dc magnetization curves. The difference in the observed energy barriers can be explained by the presence of two different modes of superparamagnetic relaxation which are characteristic of the weakly ferromagnetic phase. One mode involves a rotation of the sublattice magnetization directions in the basal (111) plane, which gives rise to superparamagnetic behavior in both Mössbauer spectroscopy and magnetization measurements. The other mode involves a fluctuation of the net magnetization direction out of the basal plane, which mainly affects the magnetization measurements.
\end{abstract}

\section{INTRODUCTION}

Hematite $\left(\alpha-\mathrm{Fe}_{2} \mathrm{O}_{3}\right)$ is the most stable iron oxide under ambient conditions and is commonly found in nature. ${ }^{1,2}$ Its magnetic properties have been studied extensively both in bulk form and in the form of ultrafine particles. ${ }^{1}$ Although many reports concerning hematite nanoparticles have appeared, see, e.g., ${ }^{1,3-19}$ the magnetic properties of the small particles are still not understood in detail.

Hematite has the corundum crystal structure and orders antiferromagnetically below its Néel temperature, $T_{N}$ $\simeq 955 \mathrm{~K}$. Bulk hematite has a Morin transition at $T_{M}$ $\simeq 263 \mathrm{~K}$ below which the two magnetic sublattices are oriented along the rhombohedral [111] axis and are exactly antiparallel. Above $T_{M}$ the moments lie in the basal (111) plane with a slight canting away from the antiferromagnetic axis resulting in a small net magnetization in the plane. For small particles the Morin temperature decreases with decreasing particle size and for particles smaller than 8-20 nm the transition temperature is below $4 \mathrm{~K}^{7-11}$ This effect has been explained by a lattice expansion in the small particles, ${ }^{9,15}$ but strain and defects may also be important. ${ }^{1,10,11}$ The magnetic moment of hematite nanoparticles in the weakly ferromagnetic (WF) state may have contributions from both the canting of the sublattice magnetization directions and from un- compensated spins ${ }^{20}$ as observed for antiferromagnetic $\mathrm{NiO}$ nanoparticles. $^{21,22}$

The magnetic anisotropy energy of magnetically ordered particles is normally assumed to be given by

$$
E(\theta)=K V \sin ^{2} \theta,
$$

where $\theta$ is the angle between the easy direction of magnetization and the magnetization vector, $K$ is the magnetic anisotropy constant and $V$ is the particle volume. In small particles the energy barrier, $K V$, which separates the two minima at $\theta=0$ and $\theta=\pi$, may be comparable to the thermal energy. This results in superparamagnetic relaxation, i.e., fluctuations of the magnetization direction among the energy minima. The temperature dependence of the superparamagnetic relaxation time, $\tau$, is expected to follow the NéelBrown expression ${ }^{23,24}$

$$
\tau=\tau_{0} \exp \left(\frac{K V}{k T}\right),
$$

where $k$ is Boltzmann's constant and $T$ is the temperature. The pre-exponential factor $\tau_{0}$ is in general of the order of $10^{-9}-10^{-12} \mathrm{~s}$ and depends weakly on temperature. ${ }^{24}$ In earlier studies of hematite nanoparticles using Mössbauer spectroscopy $y^{4,5,13,16,25,26}$ and neutron-scattering ${ }^{18}$ the analysis 
a)

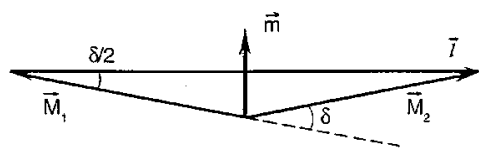

b)

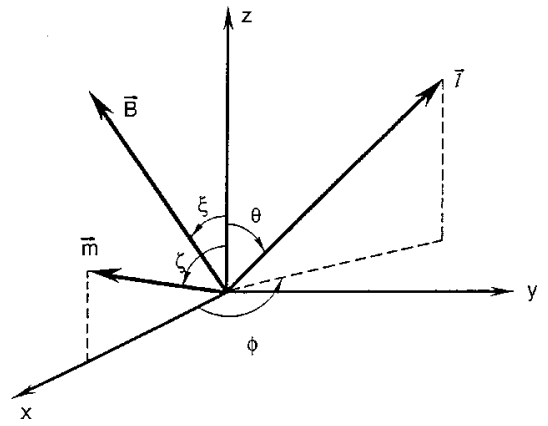

FIG. 1. (a) Illustration of the canting angle $\delta$ between two sublattice magnetizations $\mathbf{M}_{1}$ and $\mathbf{M}_{2}$, the resulting magnetization $\mathbf{m}$ $=\mathbf{M}_{1}+\mathbf{M}_{2}$, and the vector $\mathbf{l}=\mathbf{M}_{2}-\mathbf{M}_{1}$. (b) Definition of angles in a spherical polar coordinate system with the $z$ axis being the [111] orthorhombic direction.

has been based on the assumption that the superparamagnetic relaxation can be described by an expression of the same form as Eq. (2).

In this paper we present the results of a detailed study of hematite nanoparticles with a size of about $16 \mathrm{~nm}$. The particles were investigated by electron microscopy, x-ray and neutron diffraction, magnetization measurements and Mössbauer spectroscopy with the main focus on their magnetic properties. The experiments give evidence for the presence of two superparamagnetic relaxation modes as expected for hematite nanoparticles if the magnetic structure is similar to that of WF bulk hematite.

\section{THE MAGNETIC ENERGY OF WF HEMATITE}

The magnetic anisotropy energy of bulk hematite is more complex than that given by Eq. (1) because of the low symmetry of the crystal structure. The following brief summary of the magnetic properties of hematite is based on Ref. 1. The definitions of vectors and angles are illustrated in Fig. 1.

It is generally accepted that the exchange interaction in the WF state of hematite contains an anisotropic term in addition to the usual isotropic contribution. The magnetic energy per unit volume due to exchange interaction can be written

$$
F_{E}=J_{e} \mathbf{M}_{1} \cdot \mathbf{M}_{2}-\mathbf{D} \cdot\left(\mathbf{M}_{1} \times \mathbf{M}_{2}\right),
$$

where $\mathbf{M}_{1}$ and $\mathbf{M}_{2}$ are the sublattice magnetizations with $\left|\mathbf{M}_{1}\right|=\left|\mathbf{M}_{2}\right|=M, J_{e}$ is the mean-field coefficient related to the isotropic exchange interaction, and $\mathbf{D}$ is a constant vector along the [111] direction. If a magnetic field, $\mathbf{B}$, is applied it gives rise to a Zeeman energy term

$$
F_{Z}=-\mathbf{B} \cdot\left(\mathbf{M}_{1}+\mathbf{M}_{2}\right) \text {. }
$$

The magnetocrystalline anisotropy energy of bulk hematite is expected to reflect the crystal symmetry and it is therefore reasonable to assume a uniaxial contribution of the form

$$
F_{U}=-\frac{K_{1}}{2}\left(\cos ^{2} \theta_{1}+\cos ^{2} \theta_{2}\right)-\frac{K_{2}}{2}\left(\cos ^{4} \theta_{1}+\cos ^{4} \theta_{2}\right),
$$

where $K_{1}$ and $K_{2}$ are anisotropy energy constants and $\theta_{1}$ and $\theta_{2}$ are the polar angles between $\mathbf{M}_{1}$ and $\mathbf{M}_{2}$, and the [111] direction. At room temperature $\left|K_{2}\right| \ll\left|K_{1}\right|{ }^{1}$ The spin flip at the Morin temperature is related to a change in sign of $K_{1}$ from positive below $T_{M}$ to negative above $T_{M} \cdot{ }^{1}$ The sixfold symmetry in the basal (111) plane can be taken into account by introducing the following expression for the basal plane crystalline anisotropy:

$$
F_{B}=\frac{K_{B}}{2}\left(\sin ^{6} \theta_{1} \cos 6 \phi_{1}+\sin ^{6} \theta_{2} \cos 6 \phi_{2}\right),
$$

where $\phi_{1}$ and $\phi_{2}$ are the azimuthal angles of $\mathbf{M}_{1}$ and $\mathbf{M}_{2}$, respectively, and $K_{B}$ is the basal plane anisotropy energy constant. The value of $K_{B}$ is very small compared to that of $\left|K_{1}\right|$ (and $K_{2}$ ). The total magnetic energy density is given by

$$
F=F_{E}+F_{Z}+F_{U}+F_{B} .
$$

The anisotropic exchange term in Eq. (3) is responsible for the small canting of the sublattice magnetizations in the WF state. If a magnetic field is applied it will give rise to a second contribution to the canting. The resulting canting angle, $\delta$, defined in Fig. 1, is given by ${ }^{1,27}$

$$
\sin \delta \simeq \frac{1}{J_{e} M}\left[B \cos (\zeta-\xi)+D M\left(\sin ^{2} \zeta-\cos ^{2} \theta\right)^{1 / 2}\right],
$$

where $\zeta, \xi$ and $\theta$ are the angles between the [111] direction and the net magnetization $\mathbf{m}=\mathbf{M}_{1}+\mathbf{M}_{2}$, the applied field, and $\mathbf{I}=\mathbf{M}_{2}-\mathbf{M}_{1}$, respectively. In the WF state $K_{1}<0$ and $\mathbf{I}$ will therefore be in the basal plane, i.e., $\theta=\pi / 2$. In zero applied field we thus obtain the expression

$$
\sin ^{2} \delta \simeq\left(\frac{D}{J_{e}}\right)^{2} \sin ^{2} \zeta
$$

The largest canting angle $\delta_{0} \approx D / J_{e}$ is obtained when $\mathbf{m}$ is in the basal plane. Since $\delta_{0} \ll 1$ the change in uniaxial anisotropy energy during rotation of $\mathbf{m}$ out of the plane is negligible. In zero applied field, the energy required to rotate $\mathbf{m}$ an angle $\zeta$ out of the basal plane is therefore given by the change in the exchange interaction energy density [Eq. (3)], which can be written as

$$
F_{m} \simeq-K_{D} \sin ^{2} \zeta,
$$

where $K_{D}=(M D)^{2} / 2 J_{e}$. From this expression we find that for a particle with volume $V$ there is an energy barrier $K_{D} V$ for a rotation of $\mathbf{m}$ out of the (111) plane. With the values $M=9.0 \times 10^{5} \mathrm{~J} \mathrm{~T}^{-1} \mathrm{~m}^{-3}, M D=2.0 \mathrm{~T}$ and $M J_{e}=900 \mathrm{~T}$ for bulk hematite ${ }^{1,28,29}$ we estimate $K_{D}=2.0 \times 10^{3} \mathrm{~J} \mathrm{~m}^{-3}$.

When a sufficiently large magnetic field, $\mathbf{B}$, is applied to WF hematite such that $\mathbf{m}$ is aligned along the field we find from Eq. (8) that

$$
m=2 M \sin \frac{\delta}{2} \simeq \frac{1}{J_{e}}(B+D M \sin \zeta),
$$


where we have used that $0 \leqslant \zeta \leqslant \pi, \xi=\zeta, \theta=\pi / 2$ and $\delta \ll 1$. If $\mathbf{B}$ is applied in the basal plane the magnetization is given by $m=\rho\left(\sigma_{0}+\chi B\right)$, where $\rho$ is the density, $\chi=\left(\rho J_{e}\right)^{-1}$ is the observed mass susceptibility and $\sigma_{0}=D M / \rho J_{e}$ is the spontaneous magnetization per unit mass. For $B=0$ the spontaneous magnetization in the basal plane is $m_{0}=\rho \sigma_{0}$.

\section{EXPERIMENT}

The hematite nanoparticles were prepared by heating 250 g $\mathrm{Fe}\left(\mathrm{NO}_{3}\right)_{3} \cdot 9 \mathrm{H}_{2} \mathrm{O}$ (Merck, p.a.) at $90{ }^{\circ} \mathrm{C}$ in air for 20 days. The final mass of the as-prepared sample was $55.8 \mathrm{~g}$, which is $13 \%$ higher than expected for a full transformation of the iron salt to pure $\mathrm{Fe}_{2} \mathrm{O}_{3}$. This was also reflected by a thermogravimetric analysis up to $800{ }^{\circ} \mathrm{C}$, which showed a $13 \%$ decrease of the mass. The excess material is probably due to water and nitrate ions mainly associated with the interface between the particles.

A ferrihydrite impurity (see Sec. IV B) in the as-prepared sample was removed by treating the sample in darkness for $20 \mathrm{~h}$ with a solution of $0.2 \mathrm{M}$ ammonium oxalate with the $\mathrm{pH}$ adjusted to 3 using $1 \mathrm{M} \mathrm{HCl} .{ }^{30,31}$ The hematite particles were first separated from the fluid by centrifugation and then washed twice with water. Finally, the particles were coated with oleic acid and dispersed in hexadecane as described in Ref. 32. The resulting sample is labeled $C 1$.

In order to perform Mössbauer studies of fast superparamagnetic relaxation at elevated temperatures a sample of coated hematite particles supported on porous silica (Matrex Silica, $320 \mathrm{~m}^{3} \mathrm{~g}^{-1}$, Amicon Corp.) was prepared. First, the average size of the coated particles dispersed in hexadecane was reduced by removal of the largest particles by centrifugation. The suspension was added to the dry silica and the hexadecane was evaporated at reduced pressure at about 385 $\mathrm{K}$, giving a sample containing approximately $12 \mathrm{wt} . \%$ hematite on silica. This sample is labeled $C 2$. The silica was used to reduce particle interactions and to prevent sintering at elevated temperatures.

Bulk polycrystalline hematite in the form of a commercial hematite powder (Merck, p.a.) was studied as a reference. Prior to the study the material was annealed in air for $3 \mathrm{~h}$ at $1170 \mathrm{~K}$ resulting in an increase of the average particle size from about 0.5 to $3 \mu \mathrm{m}$. This sample is labeled $B$.

Neutron-diffraction measurements were performed at 10 $\mathrm{K}$ using the TAS7 triple-axis spectrometer at DR3, Ris $\varnothing \mathrm{Na}-$ tional Laboratory. Mössbauer spectroscopy was performed using constant-acceleration spectrometers with $50 \mathrm{mCi}$ sources of ${ }^{57} \mathrm{Co}$ in $\mathrm{Rh}$. The spectrometers were calibrated using a $12.5 \mu \mathrm{m}$ thick $\alpha$-iron foil at room temperature. Isomer shifts are given relative to the centroid of the calibration spectrum. Mössbauer spectra at temperatures between 5 and $230 \mathrm{~K}$ in applied magnetic fields up to $4 \mathrm{~T}$ were obtained using a liquid helium cryostat with a superconducting magnet from Thor Cryogenics. Zero-field spectra at temperatures between 15 and $300 \mathrm{~K}$ were obtained using a closed cycle helium refrigerator from APD Cryogenics Inc. Spectra at higher temperatures were obtained using a home-built furnace. ac and dc magnetization measurements were carried out using commercial superconducting quantum interference device (SQUID) magnetometers from Cryogenic Ltd. and Quantum Design, respectively. Transmission electron mi- croscopy (TEM) was performed using a Philips EM 430 microscope operated at $300 \mathrm{kV}$. X-ray diffraction (XRD) patterns were obtained using a Siemens D5000 diffractometer and $\mathrm{CoK}_{\alpha}$ radiation. Both the as-prepared sample and the samples of coated particles (after evaporation of the carrier liquid in vacuum at $385 \mathrm{~K}$ ) were studied. The iron content in the dried $C 1$ sample was measured by atomic absorption spectroscopy (AAS) and from this the weight percent of hematite was determined to $71 \pm 2 \%$. The remaining sample mass is mainly due to oleic acid surfactant and residual carrier liquid.

The Mössbauer study of sample $C 1$ was performed with the hexadecane carrier frozen in zero applied magnetic field. In order to avoid induced texture, magnetic fields were only applied at temperatures up to $230 \mathrm{~K}$, which is well below the melting point of the carrier liquid. No magnetic fields were applied to the part of sample $C 2$, which was studied by Mössbauer spectroscopy. All magnetization measurements were performed on powder samples firmly compressed in teflon cups. The dried $C 1$ sample was used in these measurements to avoid the large diamagnetic contribution from the carrier liquid. A comparison of magnetization measurements obtained before and after exposure of the $C 1$ sample to $5 \mathrm{~T}$, revealed that no texture had been induced by the field.

\section{RESULTS}

\section{A. Particle size and structure}

Figure 2(a) shows the XRD spectrum of the dried $C 1$ sample. The hematite diffraction pattern is the dominant feature with an additional broad low-intensity peak at $q$ $=1.4 \AA^{-1}$ due to the surfactant. The spectrum of the asprepared sample is very similar except that the broad peak is absent. From the line broadening of the diffraction peaks the particle size can be obtained using the Scherrer formula $d$ $=K \lambda / \beta \cos \theta$, where $\lambda$ is the wavelength and $\theta$ is the Bragg angle. Assuming a spherical particle shape the particle diameter $d$ is obtained by setting $K=1.107$ when $\beta$ is the full width at half maximum line breadth. ${ }^{33}$ A particle size of $16 \pm 3 \mathrm{~nm}$ was determined for both the as-prepared sample and sample $C 1$ assuming negligible lattice strain [a similar analysis based on the integral breadth with $K=1.333$ (Ref. 33) gave a particle size of $17 \pm 4 \mathrm{~nm}$ ]. The line broadening was also analyzed for the effect of lattice strain but no consistent effect could be resolved. A strain level of about $0.01-$ $0.03 \%$ cannot be excluded and as a result the obtained particle size may be slightly underestimated but this effect is within the stated uncertainty.

In the neutron-diffraction pattern of the as-prepared sample, shown in Fig. 2(b), the two peaks at $q=1.37 \AA^{-1}$ and $1.51 \AA^{-1}$ are the purely magnetic (111) and (100) reflections; the other lines are due to nuclear diffraction. The peaks at $q=2.69 \AA^{-1}$ and $3.10 \AA^{-1}$ are due to the aluminum sample container. The particle size and the magnetic domain sizes were determined from the broadening of the structural and magnetic peaks, respectively. Both the structural and the magnetic sizes were $15 \pm 4 \mathrm{~nm}$, in good agreement with the $\mathrm{XRD}$ results.

For both X-ray and neutron scattering the contribution to the diffraction pattern from each particle is weighted by the particle volume and the obtained particle size can thus be considered to be related to the volume-weighted average par- 


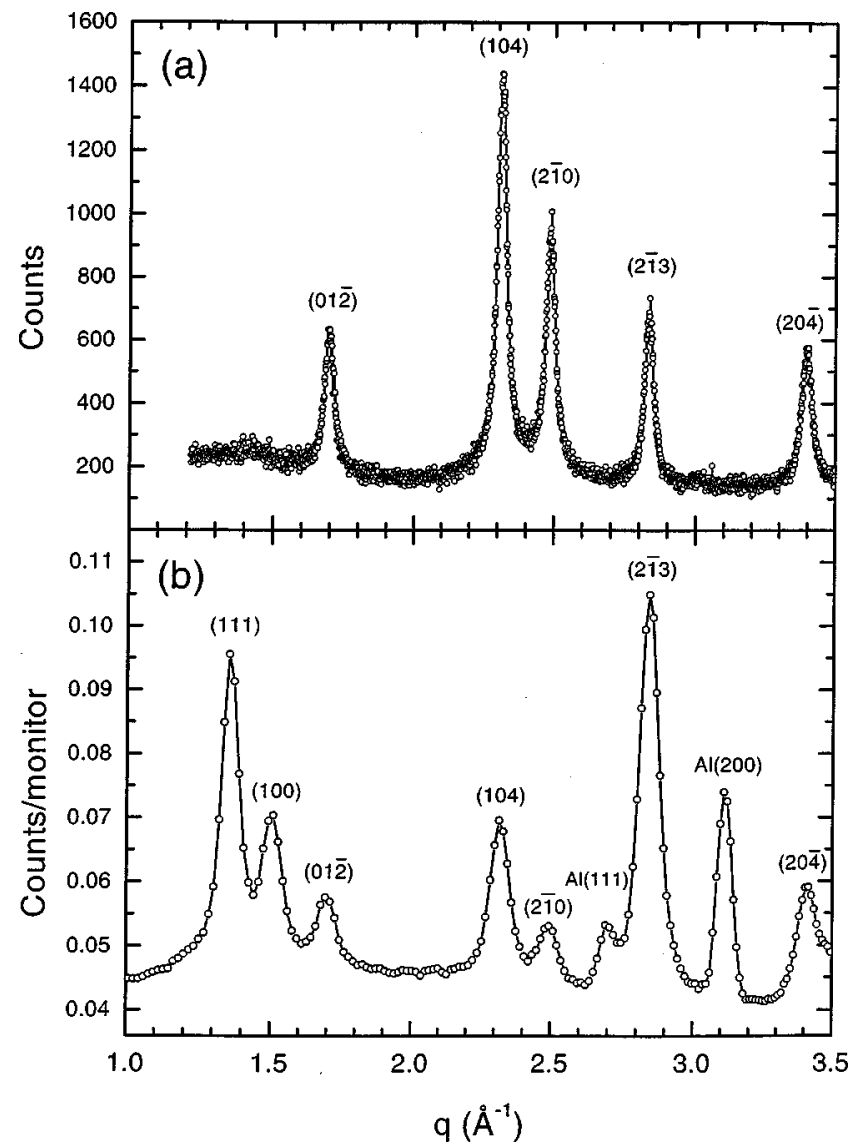

FIG. 2. (a) X-ray diffraction spectrum of the dried sample $C 1$ with the carrier liquid removed and (b) neutron-diffraction spectrum of the as-prepared sample (the solid lines are a guide to the eye). Both spectra are shown as a function of the momentum transfer $q$.

ticle size. Thus, we conclude that the particles consist of single magnetic domains with an average volume-weighted diameter of $16 \pm 3 \mathrm{~nm}$. For sample $C 2$ a particle diameter of $14 \pm 3 \mathrm{~nm}$ was estimated using XRD before impregnation of the silica.

Transmission electron micrographs of the samples $C 1$ and $C 2$ (before impregnation of the silica) showed roughly spherically shaped particles but with some irregularities and a slight degree of agglomeration, especially in sample $C 1$, and this makes a detailed estimate of the particle size distribution difficult. An estimate of the size distribution was, however, possible for sample $C 2$. Assuming spherical particle shape, the volume-weighted size distribution could be described well by a log-normal distribution with a volumeweighted median particle diameter $d_{m}=16 \pm 3 \mathrm{~nm}$ and the standard deviation $\sigma_{d}=0.22 \pm 0.09$ of $\ln \left(d / d_{m}\right)$. Thus the distribution of volume-weighted particle volumes is also lognormal distributed with the median volume $V_{m}=\pi\left(d_{m}\right)^{3} / 6$ and the standard deviation $\sigma=3 \sigma_{d}=0.7 \pm 0.3$ of $\ln \left(V / V_{m}\right)$. The average volume-weighted diameter for the log-normal distribution is $d_{\mathrm{av}}=d_{m} \exp \left(\sigma_{d}^{2} / 2\right)=d_{m} \cdot 1.02=16 \pm 3 \mathrm{~nm} .{ }^{34}$ This value is slightly larger than that found from the XRD analysis but the difference is within the uncertainty. A similar analysis of TEM micrographs of sample $C 1$ showed that, when a few agglomerates were ignored, the particle size distribution in this sample is also compatible with a log-normal distribution.

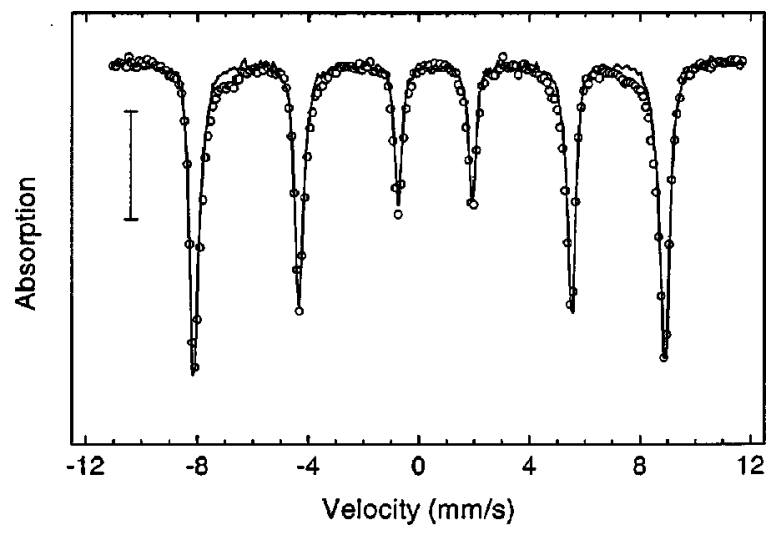

FIG. 3. Mössbauer spectrum obtained at $15 \mathrm{~K}$ of the as-prepared sample (circles) and of sample $C 1$ (shown as a solid line between data points). The spectrum of the as-prepared sample is scaled by a factor 0.54 such that absorption areas of the hematite component are the same in the spectra. The vertical bar represent $2 \%$ absorption for the sample $C 1$.

In the following we use the average particle diameters determined by the diffraction techniques when such average values are relevant. When the size distribution is important it will, however, be assumed that the size distribution for both sample $C 1$ and $C 2$ can be described by a log-normal distribution.

\section{B. Mössbauer spectra of the as-prepared sample and of sample C1}

Figure 3 shows Mössbauer spectra obtained at $15 \mathrm{~K}$ of the as-prepared sample and of sample $C 1$. The main feature of both spectra is the magnetically split sextet due to hematite with a magnetic hyperfine field of about 52.5 T. For the as-prepared sample there is an additional component with broader lines and a hyperfine field of about $48 \mathrm{~T}$, which probably is due to ferrihydrite or amorphous $\mathrm{Fe}_{2} \mathrm{O}_{3} \cdot{ }^{35}$ This component, which corresponds to about $10 \%$ of the spectral area of the as-prepared sample, is not visible in the spectra of sample $C 1$ and must therefore have been reduced to less than about $2 \%$ by the oxalate treatment. This result is in good agreement with other studies which indicate that ferrihydrite is dissolved by the oxalate treatment. ${ }^{30,31}$

Mössbauer spectra of sample $C 1$ were obtained in zero applied field at temperatures between 5 and $230 \mathrm{~K}$. Selected spectra are shown in Fig. 4. At $5 \mathrm{~K}$ the spectrum is magnetically split with a hyperfine field of $53.1 \pm 0.2 \mathrm{~T}$, an isomer shift of $0.49 \pm 0.01 \mathrm{~mm} \mathrm{~s}^{-1}$ and a quadrupole shift of $-0.102 \pm 0.006 \mathrm{~mm} \mathrm{~s}^{-1}$. These values are consistent with those expected for weakly ferromagnetic hematite with the sublattice magnetization directions in the basal plane. ${ }^{36}$ This shows that there is no Morin transition at temperatures as low as $5 \mathrm{~K}$. At this temperature, the absorption lines have a nearly Lorentzian line shape with the width $0.42 \mathrm{~mm} \mathrm{~s}^{-1}$ of lines 1 and 6 . This width is slightly larger than the corresponding $0.29 \mathrm{mms}^{-1}$ obtained for the bulk polycrystalline hematite. As the temperature is increased, there is a gradual collapse of the magnetically split component to a doublet, which is the dominant feature at $230 \mathrm{~K}$. This behavior is typical for magnetic nanoparticles, which exhibit superparamagnetic relaxation. ${ }^{4,5,37}$ The gradual collapse of the mag- 


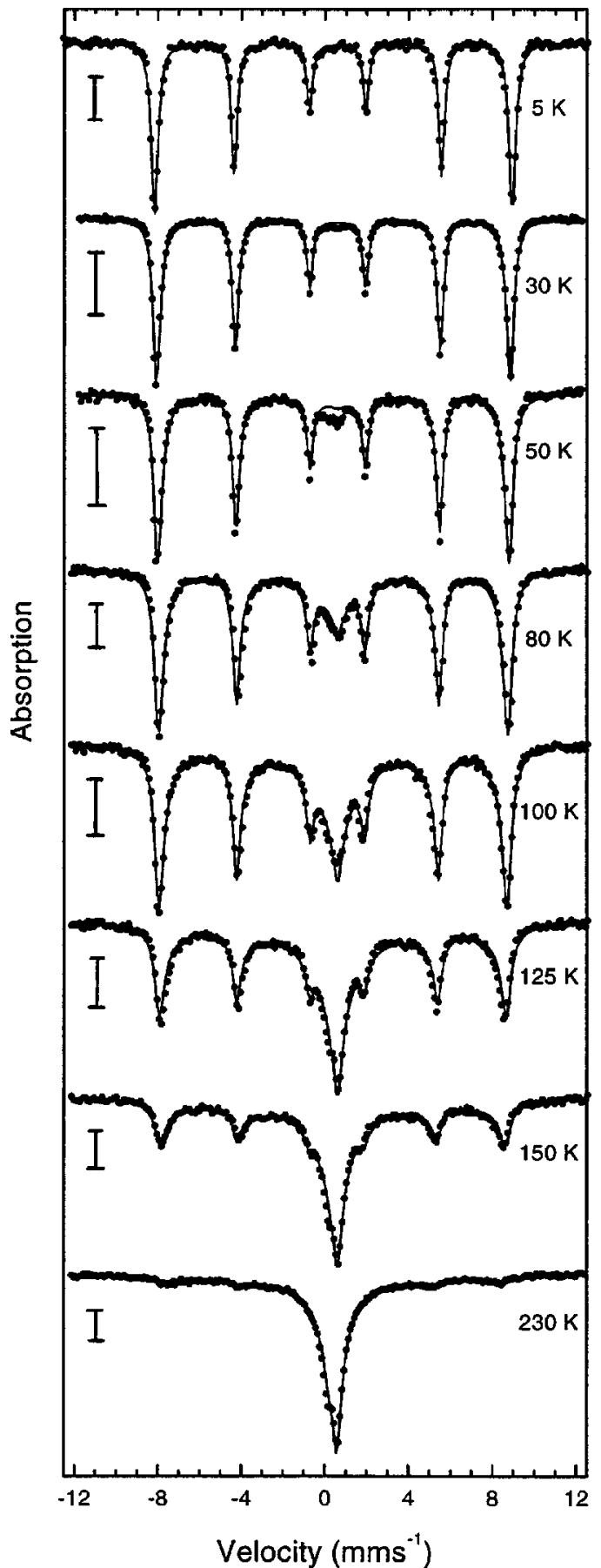

FIG. 4. Selected Mössbauer spectra of sample $C 1$ measured at the indicated temperatures. The full lines are fits based on the model described in the text. The vertical bars represent $2 \%$ absorption.

netically split spectrum with increasing temperature indicates that the sample contains particles with a broad distribution of blocking temperatures, $T_{B}$, which is related to the particle size distribution. The relative area of the magnetically split component varies almost linearly with temperature over a broad temperature range around the median blocking temperature, $T_{B m}$, defined as the temperature at which $50 \%$ of the sextet has collapsed. This analysis yields a median blocking temperature $T_{B m}=143 \pm 5 \mathrm{~K}$.

The magnetic field dependence of the spectrum of sample $C 1$ at $230 \mathrm{~K}$ is illustrated in Fig. 5. An applied field of $0.7 \mathrm{~T}$

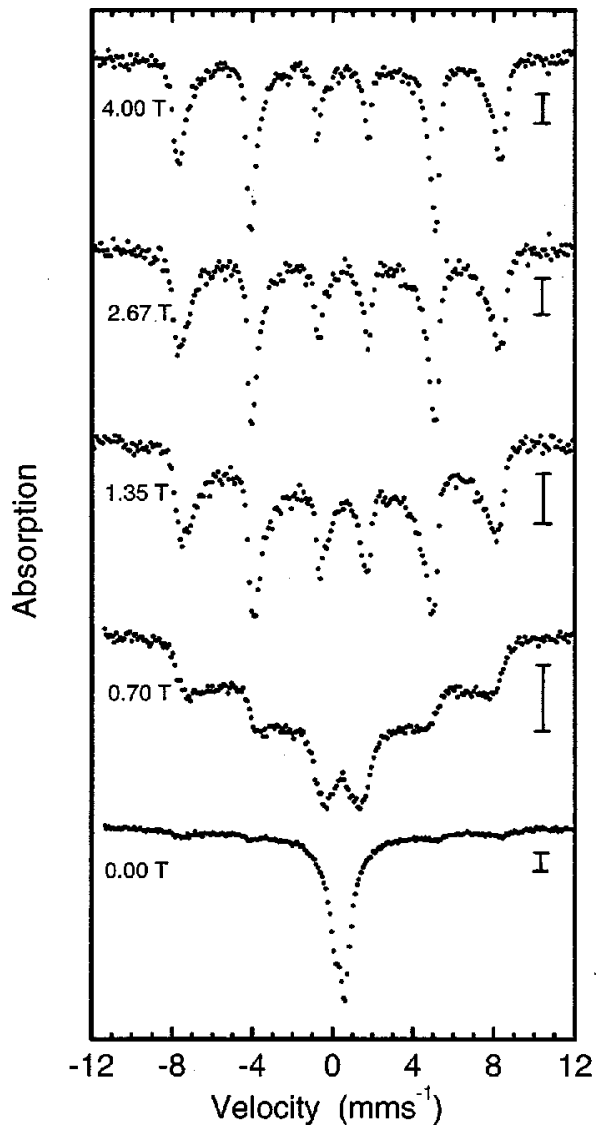

FIG. 5. Mössbauer spectra of sample $C 1$ obtained at $230 \mathrm{~K}$ in zero field and in different applied magnetic fields. The field of $0.7 \mathrm{~T}$ was applied perpendicular to the gamma-ray direction, while the larger fields were applied parallel to the gamma-ray direction. The vertical bars represent $1 \%$ absorption.

is seen to be sufficient to induce a substantial magnetic splitting. For larger applied fields the hyperfine splitting approaches the values found below the blocking temperature. This shows that the Zeeman energy is comparable to or larger than the thermal energy for $B \gtrsim 1 \mathrm{~T}$.

Mössbauer spectra of sample $C 1$ were obtained at $5 \mathrm{~K}$ in a range of magnetic fields applied parallel to the gamma-ray direction. Selected spectra are shown in Fig. 6. The main effect of the field is to change the area ratio of the absorption lines. The area ratio of the absorption lines in a magnetically

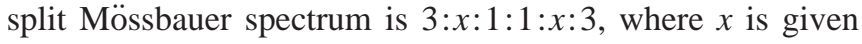
by the expression

$$
x=\frac{4 \sin ^{2} \psi}{2-\sin ^{2} \psi}
$$

and $\psi$ is the angle between the hyperfine field and the direction of the gamma-rays. The observed area ratio can therefore be used to obtain information on the orientation of the sublattice magnetizations. In zero field the lines have an area ratio close to $3: 2: 1: 1: 2: 3$ as expected for particles with a random orientation of their sublattice magnetizations. In applied fields the observed magnetic hyperfine field, $B_{\text {obs }}$, is almost unchanged, but the relative intensities of lines 2 and 5 increase with increasing magnetic field strength. The values of $x$, obtained by fitting each of the spectra to a sextet with 


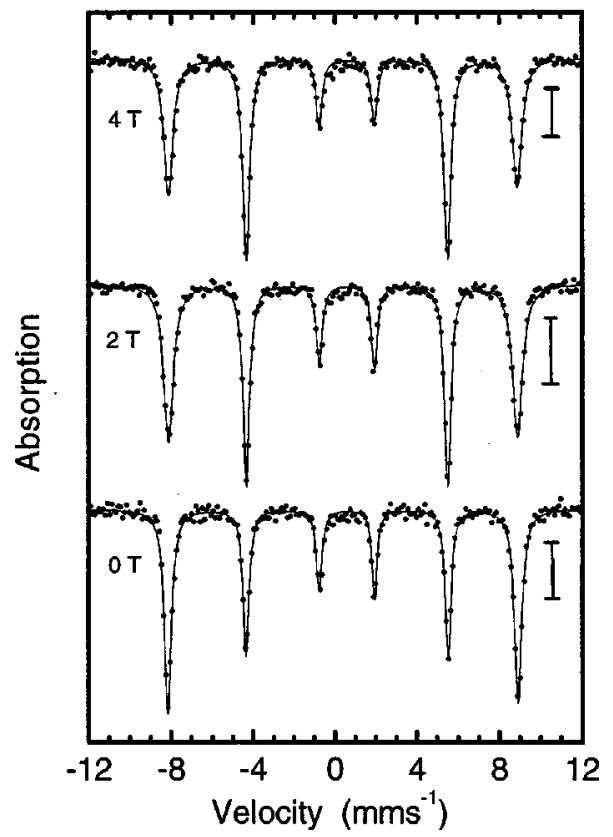

FIG. 6. Selected Mössbauer spectra of sample $C 1$ measured at 5 $\mathrm{K}$ in zero field and with different magnetic fields applied parallel to the gamma-ray direction. The vertical bars represent $2 \%$ absorption.

the area ratio $3: x: 1: 1: x: 3$ of the lines, are plotted as a function of the applied magnetic field in Fig. 7.

\section{Mössbauer spectra of sample $C 2$}

In order to study superparamagnetic relaxation well above $T_{\mathrm{Bm}}$ Mössbauer spectra of sample $C 2$ were obtained as a function of temperature up to $495 \mathrm{~K}$ in zero external field. Representative spectra, obtained at the indicated temperatures, are shown in Fig. 8. The main difference compared to sample $C 1$ is that $T_{\mathrm{Bm}}$ is slightly lower due to the smaller particle size of sample $C 2$. After the measurement at $495 \mathrm{~K}$ some of the spectra at lower temperatures were measured again and a slight increase of $T_{\mathrm{Bm}}$ was observed, but the changes of the spectra in the temperature range from 295 to $495 \mathrm{~K}$ was negligible. Thus the sample was not significantly affected by the heating. Annealing at $523 \mathrm{~K}$, however, re-

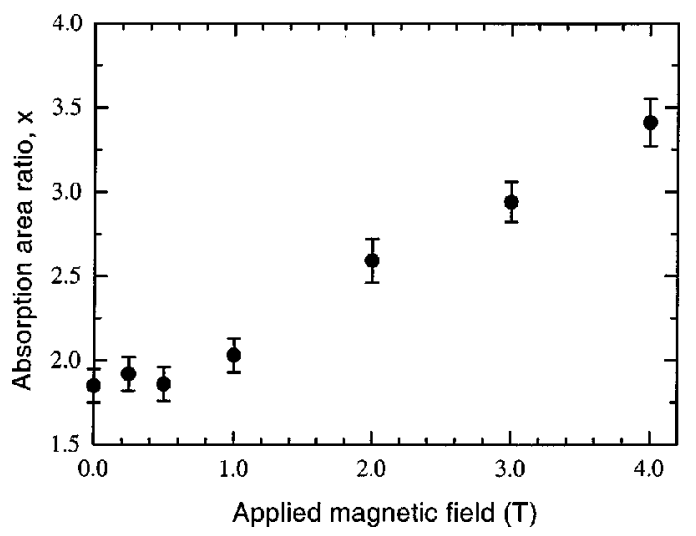

FIG. 7. Relative absorption area, $x$, of lines 2 and 5 in the magnetically split Mössbauer spectra of sample $C 1$ at $5 \mathrm{~K}$ shown as a function of the applied magnetic field.
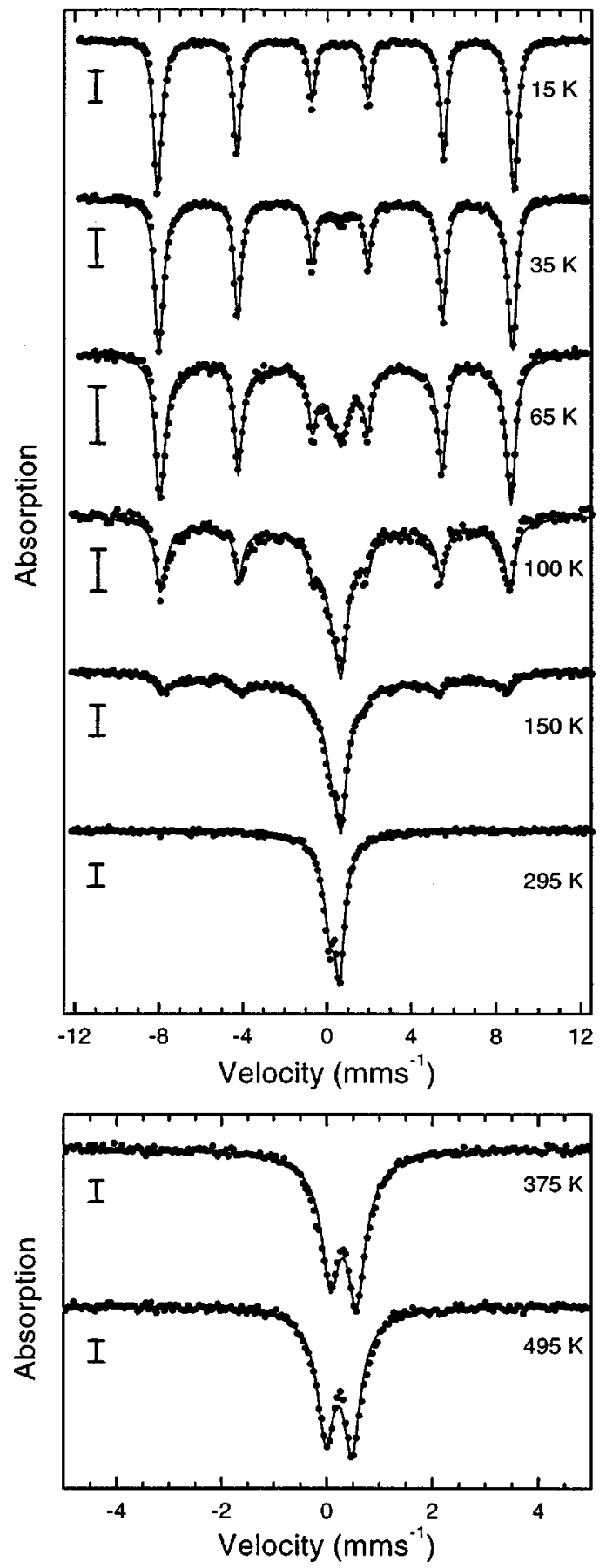

FIG. 8. Selected Mössbauer spectra of sample $C 2$ obtained at the indicated temperatures. The full lines are fits based on the model described in the text. Note the different velocity scales in the upper and lower part of the figure. The vertical bars represent $1 \%$ absorption.

sulted in a fully magnetically split Mössbauer spectrum at $295 \mathrm{~K}$, indicating that some sintering of the particles had taken place.

Because of the lower blocking temperature of sample $C 2$ the spectra obtained at temperatures above $200 \mathrm{~K}$ consist essentially of a superparamagnetic doublet. This doublet is asymmetric with different widths but equal absorption areas of the two lines. As the temperature is increased the asymmetry is reduced and by repeating the measurements at lower temperatures it was shown that this effect is reversible. 


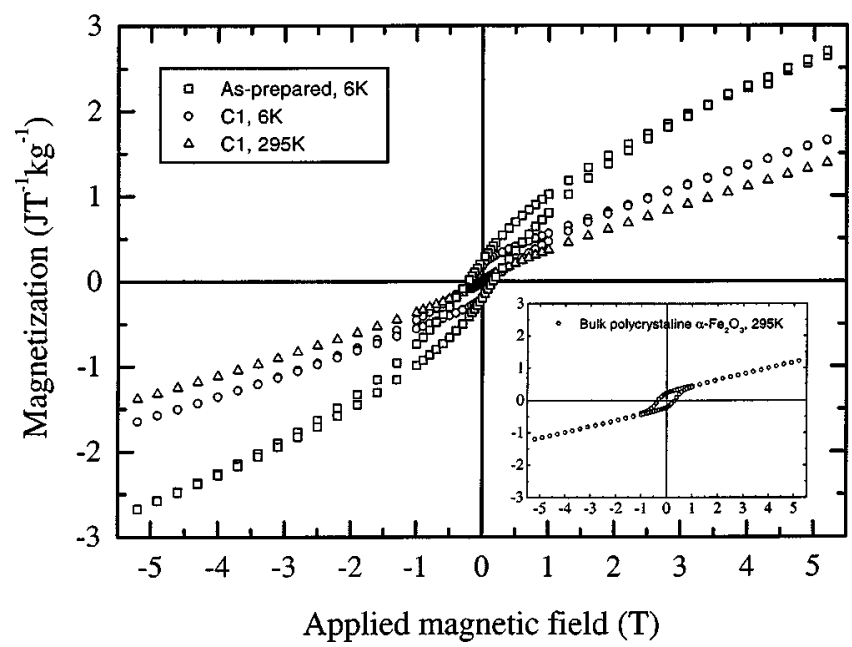

FIG. 9. Hysteresis loop of the as-prepared sample ( $\square)$ and of the dried sample $C 1$ at $6 \mathrm{~K}(\bigcirc)$ and $295 \mathrm{~K}(\triangle)$. The magnetization is given per unit mass of $\mathrm{Fe}_{2} \mathrm{O}_{3}$ in the sample as determined by AAS. The inset shows the hysteresis loop of sample $B$ measured at $295 \mathrm{~K}$.

\section{Magnetization measurements}

Figure 9 shows the hysteresis curves for sample $C 1$ measured at 6 and $295 \mathrm{~K}$ and for the as-prepared sample measured at $6 \mathrm{~K}$. The inset shows the corresponding curve for sample $B$ measured at $295 \mathrm{~K}$. The measurements indicate that the hysteresis loops are closed for magnetic fields larger than about $4 \mathrm{~T}$. The high-field differential magnetic mass susceptibilities, $\chi$, were determined from the slopes of the linear high-field parts of the hysteresis curves after correction for diamagnetic contributions from the sample holder. The spontaneous magnetization, $\sigma_{s}$, was found by extrapolation of the linear part of the high-field magnetization to zero field. The results are given in Table I where the results obtained for sample $C 2$ at $10 \mathrm{~K}$ and $295 \mathrm{~K}$ are also given. The data obtained at $295 \mathrm{~K}$ and at low temperatures cannot be directly compared because superparamagnetic relaxation has a significant influence on the magnetization at $295 \mathrm{~K}$ even for $B$ $=4 \mathrm{~T}$, whereas the influence of superparamagnetism is negligible at low temperatures in large applied fields. The coercive field of sample $C 1$ was $0.15 \mathrm{~T}$ at $6 \mathrm{~K}$. It was found to

TABLE I. The spontaneous magnetization, $\sigma_{s}$, and the highfield susceptibility, $\chi$, of the polycrystalline samples and of bulk single-crystal hematite measured in the basal plane. The data for bulk single crystals are taken from Ref. 28.

\begin{tabular}{lccc}
\hline \hline Samples & $\begin{array}{c}\text { Temperature } \\
(\mathrm{K})\end{array}$ & $\begin{array}{c}\sigma_{s} \\
\left(\mathrm{~J} \mathrm{~T}^{-1} \mathrm{~kg}^{-1}\right)\end{array}$ & $\begin{array}{c}\chi \\
\left(\mathrm{J} \mathrm{T}^{2} \mathrm{~kg}^{-1}\right)\end{array}$ \\
\hline As-prepared & 6 & $0.99 \pm 0.09$ & $0.34 \pm 0.04$ \\
& 295 & $0.21 \pm 0.03$ & $0.27 \pm 0.04$ \\
$C 1$ (dried) & 6 & $0.40 \pm 0.03$ & $0.27 \pm 0.03$ \\
& 150 & $0.29 \pm 0.03$ & $0.26 \pm 0.03$ \\
& 295 & $0.22 \pm 0.03$ & $0.25 \pm 0.03$ \\
C2 (dried) & 10 & $0.42 \pm 0.03$ & $0.25 \pm 0.03$ \\
& 295 & $0.17 \pm 0.03$ & $0.23 \pm 0.03$ \\
$B$ & 295 & $0.29 \pm 0.02$ & $0.19 \pm 0.02$ \\
Bulk, single crystals & 295 & $0.38 \pm 0.04$ & $0.19 \pm 0.02$ \\
\hline \hline
\end{tabular}

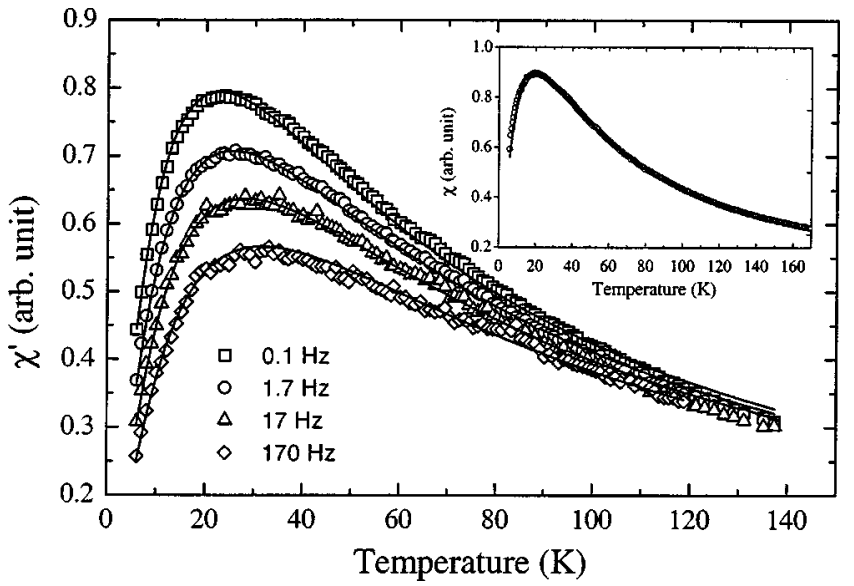

FIG. 10. The real part of the ac magnetization curves measured at the frequencies $f=0.1,1.7,17$, and $170 \mathrm{~Hz}$ after cooling in zero field. The inset shows the ZFC magnetization curve measured with an applied field of $2.0 \mathrm{mT}$. The solid lines are fits to the data based on the models described in the text.

decrease with increasing temperature and reached zero at about $100 \mathrm{~K}$. The coercive field of sample $B$ was $0.33 \mathrm{~T}$ at $295 \mathrm{~K}$.

Figure 10 shows the zero-field cooled (ZFC) magnetization curve measured after a wait time, $t_{m} \simeq 100 \mathrm{~s}$, at each temperature in an applied field of $2.0 \mathrm{mT}$ and the ac magnetization curves measured at the frequencies $f=0.1,1.7,17$, and $170 \mathrm{~Hz}$. At low temperatures the magnetic moments are frozen in an easy direction of magnetization giving rise to a low magnetic susceptibility and at high temperatures the susceptibility decreases as $T^{-1}$ due to the thermal fluctuations of the magnetic moments. The magnetic susceptibility has a peak at a temperature between these two extreme situations, where the time scale of the superparamagnetic fluctuations becomes comparable to the time scale of the measurement. It is seen from Fig. 10 that the temperature corresponding to the peak position increases with increasing frequency and that the height of the peak of the in-phase component of the ac-susceptibility decreases with increasing frequency as expected for an ensemble of non-interacting superparamagnetic particles.

\section{DISCUSSION}

\section{A. The spontaneous magnetization of sample $B$}

The value of $\sigma_{s}$ for sample $B$ is lower than the value $\sigma_{0}$ $=m_{0} / \rho=0.38 \pm 0.04 \mathrm{~J} \mathrm{~T}^{-1} \mathrm{~kg}^{-1}$ measured in the basal plane of bulk single crystals. ${ }^{28}$ This is due to the orientation dependence of the canting angle [see Eq. (9)]. If the applied magnetic field is sufficiently large to align the effective particle moment along the field direction we get from Eq. (11) the relation $\sigma(\mathbf{B})=\sigma_{0} \sin \zeta+\chi B$. For a polycrystalline bulk hematite powder we find the average magnetization by integrating over all the possible orientations of $\mathbf{B}$ relative to the [111] axis:

$$
\langle\sigma(\mathbf{B})\rangle=\frac{\pi}{4} \sigma_{0}+\chi B .
$$


This shows that we should expect an effective spontaneous magnetization of $\sigma_{s}=\pi \sigma_{0} / 4=0.30 \pm 0.03 \mathrm{~J} \mathrm{~T}^{-1} \mathrm{~kg}^{-1}$ in good agreement with the observed value, $\sigma_{s}=0.29$ $\pm 0.01 \mathrm{~J} \mathrm{~T}^{-1} \mathrm{~kg}^{-1}$, for sample $B$.

\section{B. Determination of the effective magnetic moment of the nanoparticles}

The values of $\sigma_{0}$ and $\chi$ for the hematite nanoparticles at 6 $\mathrm{K}$ can be compared to the bulk values at room temperature since the temperature dependence of these values is small in the WF phase below $300 \mathrm{~K}^{38}$ The magnetization measurements at $6 \mathrm{~K}$ show that the values of $\chi$ and $\sigma_{s}$ for sample $C 1$ are about $40 \%$ larger than the values measured for sample $B$.

The much larger value, $\sigma_{s}=0.99 \mathrm{~J} \mathrm{~T}^{-1} \mathrm{~kg}^{-1}$ (see Table I), for the as-prepared sample must be due to the presence of poorly crystalline $\mathrm{Fe}_{2} \mathrm{O}_{3}$ or ferrihydrite, which was removed by the oxalate treatment when preparing sample $C 1$. The observations can be explained if this impurity phase has a spontaneous magnetization of about $6 \mathrm{JT}^{-1} \mathrm{~kg}^{-1}$, which indeed is of the same order of magnitude as the reported value for ferrihydrite. ${ }^{39,40}$ At room temperature the magnetization values for the as-prepared and the $C 1$ samples are quite similar. This suggests that the contribution from the disordered iron-containing compound at this temperature is strongly reduced. This is also in accordance with the expected behavior of ferrihydrite. ${ }^{40}$ The value of $\sigma_{s}$ for sample $C 1$ at $6 \mathrm{~K}$ can only be considered as an upper limit for pure hematite nanoparticles as up to $2 \%$ of the iron may still be in the form of such a disordered phase, i.e., the value of $\sigma_{s}$ for the hematite nanoparticles is in the range $0.3-0.4 \mathrm{~J} \mathrm{~T}^{-1} \mathrm{~kg}^{-1}$. As the value of $\chi$ for the as-prepared sample is only slightly enhanced relative to sample $C 1$ it follows that the high-field susceptibility of the impurity compound is not much different from that of hematite.

Muench et al. ${ }^{12}$ found for 20-30 nm hematite particles a five times enhancement of the susceptibility compared to the bulk value. There are several reports on iron oxide particles with sizes of 2-10 nm which by the authors were identified as hematite with relatively large $\sigma_{s}$-values of 4-10 $\mathrm{J} \mathrm{T}^{-1} \mathrm{~kg}^{-1}$ (Refs. 14, 16, and 17) or even larger. ${ }^{13}$ These values were explained by uncompensated surface spins ${ }^{14,17}$ or lattice defects, ${ }^{13}$ but they are also of the same order of magnitude as the magnetization of the component, which was removed by the oxalate treatment in the present study. Our results show that small amounts of impurity phases may have a significant influence on the magnetization and therefore some of the previously reported values may not be representative for pure hematite.

The spontaneous magnetization $\sigma_{s}$ can be related to an effective spontaneous particle moment of $\mu_{s}=\rho V \sigma_{s}$. Calculating $V$ from the particle diameter of $16 \mathrm{~nm}$ determined from the XRD analysis and assuming that the particles are spherical with the density of bulk hematite ( $\rho$ $=5256 \mathrm{~kg} \mathrm{~m}^{-3}$ ), we find from the $6 \mathrm{~K}$ value of $\sigma_{s}$ an effective spontaneous particle moment of $\mu_{s} \approx 500 \mu_{B}$ for sample $C 1$. The uncertainty on this value is relative large due to the uncertainty on the particle size and the possibility that the sample contains a few percent ferrihydrite.

An alternative procedure to determine the magnetic moment of nanoparticles is by using the field dependence of
Mössbauer spectra at a temperature, where all the particles are superparamagnetic. When the Zeeman energy is large compared to the anisotropy energy and the thermal energy, $k T$, the induced magnetic field at the iron nucleus, $\mid \mathbf{B}_{\mathrm{obs}}$ $-\mathbf{B} \mid$, is given by the approximation ${ }^{37,41,42}$

$$
\left|\mathbf{B}_{\mathrm{obs}}-\mathbf{B}\right| \simeq B_{0}\left(1-\frac{k T}{\mu B}\right),
$$

where $B_{0}$ is the saturation hyperfine field. In a large magnetic field, where $\mathbf{m}$ is aligned with $\mathbf{B}$ (see Sec. VC), the sublattice magnetizations will be nearly perpendicular to the field and therefore $\left|\mathbf{B}_{\text {obs }}-\mathbf{B}\right| \approx B_{\text {obs }}$ is a good approximation. In lower fields, where $\mathbf{m}$ is not fully aligned with the external field, the same approximation applies since the effect of $\mathbf{B}$ is to enhance the magnetic field for one sublattice but decrease it for the other, resulting in line broadening without any significant effect on the average hyperfine field. In the present case the magnetic moment of the particles depends on the applied field and in the high-field limit it can be expressed as

$$
\mu=\rho V\left(\sigma_{s}+\chi B\right)=\mu_{s}+\rho V \chi B .
$$

Inserting this in Eq. (14) we obtain

$$
B_{\mathrm{obs}} \simeq B_{0}\left(1-\frac{k T}{\mu_{s} B+\rho V \chi B^{2}}\right) .
$$

The Mössbauer spectra obtained at $230 \mathrm{~K}$ with applied magnetic fields in the range from 2 to $4 \mathrm{~T}$ were fitted with 2 or 3 sextets and $B_{\text {obs }}$ was determined as the area weighted average value of the hyperfine fields. The variation of $B_{\text {obs }}$ as a function of $B$ was fitted to Eq. (16) assuming a single particle size $(16 \mathrm{~nm})$ and using $\chi=0.26 \mathrm{~J} \mathrm{~T}^{-1} \mathrm{~kg}^{-1}$, obtained from the magnetization measurements at $150 \mathrm{~K}$. The fit yielded $B_{0}=50.5 \pm 0.9 \mathrm{~T}$ and $\mu_{s}=400 \pm 200 \mu_{B}$. The uncertainty on $\mu_{s}$ is mainly due to the uncertainty on $V$ in Eq. (16).

\section{The influence of uncompensated spins on the properties of sample $C 1$}

Antiferromagnetic nanoparticles are expected to have a magnetic moment due to uncompensated spins. Néel ${ }^{20}$ has proposed different models which predict uncompensated magnetic moments given by $\mu_{\mathrm{uc}}=n^{z} \mu_{\text {atom }}$, where $\mu_{\text {atom }}$ is the atomic moment (about $4.9 \mu_{B}$ for iron atoms in hematite $^{29}$ ) and $n$ is the number of magnetic atoms per particle. Depending on the model $z$ may have values of $1 / 3,1 / 2$, or $2 / 3$. The uncompensated magnetic moment is expected to be parallel to one of the sublattice magnetization directions. For $16 \mathrm{~nm}$ hematite particles we obtain uncompensated moments of about 220,1400, and $9500 \mu_{B}$ for the three $z$ values. As a spontaneous particle moment of about $400-500 \mu_{B}$ has been determined, the actual value of $z$ must be smaller than $1 / 2$. Studies of $\mathrm{NiO}$ particles suggest that $z$ is about $1 / 3{ }^{21}$ In addition there may be a contribution $\boldsymbol{\mu}_{\text {surf }}$ to the magnetic moment from disordered surface spins. This contribution can have any direction relative to the other contributions.

The total magnetic moment is given by $\boldsymbol{\mu}=\boldsymbol{\mu}_{\mathrm{WF}}+\boldsymbol{\mu}_{\mathrm{uc}}$ $+\boldsymbol{\mu}_{\text {surf }}$, where $\boldsymbol{\mu}_{\mathrm{WF}}=\mathbf{m} V$ is the magnetic moment due to the weakly ferromagnetic canting of the sublattice magnetizations. At small applied magnetic fields the sublattice magne- 
tization directions and the direction of $\boldsymbol{\mu}$ are mainly determined by the magnetic anisotropy. For larger applied magnetic fields the direction of the sublattice magnetization will be determined by the competition between the anisotropy energy and the Zeeman energy and will of course also depend on the relative size of the contributions to $\boldsymbol{\mu}$. If $\boldsymbol{\mu}_{\text {surf }}$ is small compared to $\boldsymbol{\mu}_{\mathrm{WF}}$ and $\boldsymbol{\mu}_{\mathrm{uc}}$ the magnetic moment will be composed of the two perpendicular contributions $\boldsymbol{\mu}_{\mathrm{WF}}$ and $\boldsymbol{\mu}_{\mathrm{uc}}$. In large magnetic fields the sublattice magnetization directions will approach the field direction for $\mu_{\mathrm{uc}} \gg \mu_{\mathrm{WF}}$, whereas they will tend to be perpendicular to $\mathbf{B}$ for $\mu_{\mathrm{uc}}$ $\ll \mu_{\mathrm{WF}}$. Since $\mu_{\mathrm{WF}}$ increases with increasing applied field [see Eq. (11)] it is in principle possible that the preferred direction of the sublattice magnetization can be close to the direction of the applied field at moderate field strengths but may become nearly perpendicular to the field direction at large field strengths. In this case the value of $x$, found from Mössbauer spectra obtained in magnetic fields applied along the direction of the $\gamma$ rays, should initially decrease to values below 2.0 for low field strengths but then increase and approach a saturation value close to 4.0 for large field strengths [see Eq. (12)]. The experimental data in Fig. 7 show for $B \leq 1 \mathrm{~T}$ that the value of $x$ is close to 2.0 as expected for a random orientation of the sublattice magnetizations. For larger magnetic fields the value of $x$ increases up to about 3.4 for $B=4 \mathrm{~T}$ showing that the particle moment is nearly perpendicular to the sublattice magnetizations. This indicates that $\mu_{\mathrm{WF}}$ is the dominating contribution to the particle moment. Numerical analyses of the Mössbauer and magnetization data show that $\mu_{\mathrm{uc}} \leqslant 350 \mu_{B}$ and that even in zero applied magnetic field the moment due to canting of the sublattices is comparable to or larger than the uncompensated moment (as the two contributions to $\boldsymbol{\mu}$ are perpendicular).

Further information about the direction of the sublattice magnetization can be obtained from the quadrupole shift of the magnetically split Mössbauer spectra. In hematite the principal axis of the electric field gradient at the iron nucleus is along the [111] axis. The quadrupole shift, observed in the magnetically split Mössbauer spectrum, is given by

$$
\varepsilon=\frac{\varepsilon^{\prime}}{2}\left(3 \cos ^{2} \theta-1\right),
$$

where $\theta$ is the angle between this axis and the magnetic hyperfine field and $\varepsilon^{\prime}$ is the quadrupole interaction strength. For WF hematite the value $\varepsilon=-\varepsilon^{\prime} / 2$ is obtained as $\theta=\pi / 2$. The value of $\varepsilon^{\prime}$ is about $0.21 \mathrm{mms}^{-1}$ for bulk hematite. ${ }^{43}$ From the Mössbauer spectrum of sample $C 1$, measured at 5 $\mathrm{K}$ in zero applied field, we find $\varepsilon=-0.102$ $\pm 0.006 \mathrm{~mm} \mathrm{~s}^{-1}$, which shows that the sublattice magnetization directions are close to the basal (111) plane. Within the uncertainty no change was observed in the quadrupole shift for the $5 \mathrm{~K}$ spectra in magnetic fields of up to $4 \mathrm{~T}$. Thus, the uniaxial anisotropy energy constant, $K_{1}$, for the nanoparticles is so large that the sublattice magnetization directions remain in the (111) plane even in large magnetic fields. This shows that $\left|K_{1}\right|$ is much larger than $K_{B}$ and $K_{D}$. A similar behavior has been observed for WF bulk hematite. ${ }^{44}$

Assuming that the WF hematite moment is the only contribution to $\sigma_{s}$, the magnetization in the basal plane is $\sigma_{0}$
$=4 \sigma_{s} / \pi=0.50 \pm 0.04 \mathrm{~J} \mathrm{~T}^{-1} \mathrm{~kg}^{-1}$ for sample $C 1$. With this assumption we can calculate the associated canting angle as $\delta_{0}=2 \sin ^{-1}\left(\rho \sigma_{0} / 2 M\right)=0.17 \pm 0.01^{\circ}$. The corresponding value for bulk hematite is $\delta=0.13 \pm 0.01^{\circ} .{ }^{1}$ The enhancement of the spontaneous magnetization can, however, also be accounted for if the uncompensated particle moment or the moment due to disordered surface spins is not negligible or the sample contains up to $2 \%$ ferrihydrite.

\section{Superparamagnetic relaxation and the magnetic anisotropy energy}

The magnetic anisotropy of WF hematite nanoparticles is more complicated than that of bulk hematite. Ideally, the sixfold crystal symmetry in the basal plane should result in six minima for the magnetic energy [see Eq. (6)], but the value $T_{\mathrm{Bm}}=143 \pm 5 \mathrm{~K}$, observed by Mössbauer spectroscopy, is too large to be accounted for by the weak in-plane magnetocrystalline anisotropy of bulk WF hematite $\left(K_{B}\right.$ $\left.\sim 1 \mathrm{~J} \mathrm{~m}^{-3}\right){ }^{1}$ This suggests that there are other and larger contributions to the anisotropy in the basal plane. The magnetoelastic anisotropy originating from stress will usually dominate the magnetocrystalline anisotropy except for very carefully prepared and mounted bulk single crystals. ${ }^{1}$ This contribution to the anisotropy is uniaxial in the basal plane ${ }^{1}$ and can be large enough to dominate other contributions from, for example, shape and surface anisotropy in nanoparticles. We therefore assume that the magnetocrystalline contributions $F_{U}$ and $F_{B}$ to the energy density for bulk hematite in Eqs. (5) and (6) can be replaced by

$$
F_{\mathrm{UB}}=-K_{1} \cos ^{2} \theta+K_{\mathrm{Bu}} \sin ^{2} \theta \sin ^{2} \phi,
$$

where $\phi \approx \phi_{2}+\phi_{0} \approx \pi-\phi_{1}+\phi_{0}$ and $\phi_{0}$ is the angle between a crystal axis and an easy uniaxial axis in the basal plane. $K_{\mathrm{Bu}}$ is the effective uniaxial anisotropy constant in the basal plane. In the previous section it was shown that $\theta \approx \pi / 2$.

The energy barrier for rotation of $\mathbf{m}$ out of the basal plane is still found from Eq. (10) as $K_{D} V$. It should be stressed that the rotation of $\mathbf{m}$ out of the basal plane only involves very small changes of $\theta$ and $\phi$. The change of $F_{\mathrm{UB}}$ during such a rotation is therefore negligible compared to $K_{D} V$. This type of rotation will give rise to superparamagnetic behavior in magnetization measurements but not in Mössbauer spectroscopy since the fluctuations of the sublattice magnetization directions are negligible. Another type of rotation, which will give rise to superparamagnetic behavior in both magnetization measurements and Mössbauer spectroscopy, involves a $180^{\circ}$ rotation of the sublattice magnetizations in the basal plane over the energy barrier $K_{\mathrm{Bu}} V$. If $K_{\mathrm{Bu}} V$ is different from $K_{D} V$ we then expect to observe different energy barriers by Mössbauer spectroscopy and magnetization measurements. In magnetization measurements we expect to see a cross-over from two-dimensional relaxation of $\mathbf{m}$ over the lower of the energy barriers at low temperatures to a more isotropic relaxation of $\mathbf{m}$ at higher temperatures. The first and second types of rotation correspond to the high- and low-frequency modes, respectively, observed in electron magnetic resonance measurements on the weakly ferromagnetic state of hematite. ${ }^{1}$ 


\section{E. Fitting of the dc and ac magnetization measurements}

In the analysis of the dc and ac magnetization measurements it is not feasible to take accurately into account the two energy barriers, the dependence of the canting angle on the direction of $\mathbf{m}$ relative to the basal plane and the possible presence of an uncompensated magnetic moment. We have therefore, as a first approximation, assumed that the dc and ac magnetization measurements can be modeled using a three-dimensional relaxation model and an effectively uniaxial anisotropy energy. We have furthermore assumed that the relaxation time is adequately described by the NéelBrown expression [Eq. (2)]. In the following we will consider a volume-weighted distribution $f(y)$ of reduced particle volumes or, equivalently, energy barriers, $y \equiv V / V_{m}$ $=E_{b} / E_{\mathrm{bm}}$, where $V_{m}$ and $E_{\mathrm{bm}}$ are the median particle volume and energy barrier, respectively. Following Wohlfarth ${ }^{45}$ we write the ZFC magnetic susceptibility as

$$
\chi_{\mathrm{ZFC}}\left(T, t_{m}\right) \propto \frac{m_{1}^{2}}{3 K}\left[\frac{E_{\mathrm{bm}}}{k T} \int_{0}^{T / T_{\mathrm{bm}}} y f(y) d y+\int_{T / T_{\mathrm{bm}}}^{\infty} f(y) d y\right],
$$

where $m_{1}$ is the magnetization in the absence of dynamic effects, $T_{\mathrm{Bm}} \equiv E_{\mathrm{bm}} /\left[k \ln \left(t_{m} / \tau_{0}\right)\right], t_{m}$ is the measuring time and $K$ is an effective anisotropy constant. The first contribution is from the superparamagnetic particles and the second contribution is from the blocked particles. Following Gittleman ${ }^{46}$ we write the ac susceptibility as

$$
\begin{aligned}
\chi_{\mathrm{AC}}(T, \omega) \propto & \frac{m_{1}^{2}}{3 K}\left[\frac{E_{\mathrm{bm}}}{k T} \int_{0}^{\infty} y(1+i \omega \tau)^{-1} f(y) d y+i \omega\right. \\
& \left.\times \int_{0}^{\infty} \tau(1+i \omega \tau)^{-1} f(y) d y\right]
\end{aligned}
$$

where $\omega=2 \pi f$ is the angular frequency of the applied ac field and $\tau=\tau_{0} \exp \left(y \cdot E_{\mathrm{bm}} / k T\right)$. The first contribution is from the susceptibility of the superparamagnetic particles and the second contribution is from the susceptibility of the frozen particle moments. The real and imaginary parts, $\chi_{\mathrm{AC}}^{\prime}(T, \omega)$ and $\chi_{\mathrm{AC}}^{\prime \prime}(T, \omega)$ of $\chi_{\mathrm{AC}}(T, \omega)$ are the in-phase and out-of-phase components of the measured susceptibility, respectively. The effective median energy barrier, denoted $E_{\mathrm{bm}}^{\mathrm{magn}}$, represents the combined energy barriers and the effect of a possible cross-over between relaxation in two and three dimensions. $E_{\mathrm{bm}}^{\mathrm{magn}}$ may therefore not be directly related to any of the energy barriers. For this reason we also expect that the distribution function $f(y)$, obtained from fits of the experimental data to Eqs. (19) and (20), may be wider than the actual volume distribution. We have implicitly assumed that the distribution of effective energy barriers is independent of the measuring time. If this or the assumption of a relaxation time given by Eq. (2) is not valid, it may be seen as poor fit quality for some of the frequencies.

In the following we consider a log-normal distribution of volume-weighted energy barriers,

$$
f(y) d y=\frac{1}{\sqrt{2 \pi} \sigma y} \exp \left(-\frac{\ln ^{2} y}{2 \sigma^{2}}\right) d y,
$$

where $y=E_{b} / E_{\mathrm{bm}}$ and $\sigma$ is the logarithmic standard deviation. We have performed simultaneous least-squares fits of all the magnetization data shown in Fig. 10, both with $\tau_{0}$ as a free parameter and for different fixed values of $\tau_{0}$ between $1 \times 10^{-12} \mathrm{~s}$ and $5 \times 10^{-10} \mathrm{~s}$. The variation of $\chi^{2}$ of the fit as a function of $\ln \tau_{0}$ was close to parabolic and gave an estimate of the uncertainty on the value of $\tau_{0}$. In the following all stated uncertainties are estimated from the values of $\tau_{0}$ where $\chi^{2}$ of the fit has increased $10 \%$ compared to the value in the minimum.

The best fits of the ZFC and ac magnetization data obtained for sample $C 1$ to Eqs. (19) and (20), respectively, are shown as the full lines in Fig. 10. The out-of-phase data of the ac susceptibility were included in the fitting, but are not shown in the figure. Although the experimental results are influenced by two relaxation processes with different energy barriers (and possibly different values of $\tau_{0}$ ) the measured data are fitted well by the simple model presented above. The resulting parameters are $\tau_{0}=1.8_{-1.3}^{+3.2} \times 10^{-11} \mathrm{~s}, \quad E_{\mathrm{bm}}^{\mathrm{magn}} / k$ $=305 \pm 20 \mathrm{~K}$ and $\sigma=1.00 \pm 0.01$. The stated values and uncertainties of $E_{\mathrm{bm}}^{\mathrm{magn}}$ and $\sigma$ do not take into account the simplifications made above and should therefore not be taken as representations of the actual barrier distribution. The larger values of $\tau_{0}$ correspond to the lower values of $E_{\mathrm{bm}}^{\mathrm{magn}}$. No low-field magnetization measurements were performed on sample $C 2$.

\section{F. Fitting of the Mössbauer spectra}

The temperature series of Mössbauer spectra were analyzed using the two-level relaxation model by Blume and Tjon $^{47}$ in which the magnetic hyperfine field is assumed to switch with an average frequency $\tau^{-1}$ between the values $\pm B_{\text {obs }}$ perpendicular to the principal axis of the electric field gradient. The two-level model is justified for uniaxial anisotropy and $k T \lesssim 0.3 \cdot K V$ (see, e.g., Ref. 48 , Fig. 2 ). If the value of $\tau_{0}$ is sufficiently small $\left(\lesssim 10^{-10} \mathrm{~s}\right.$ ) this condition is fulfilled in the vicinity of the blocking temperature. The line shape of the superparamagnetic doublet, observed above the blocking temperature, will therefore only differ slightly from that generated by the two-level model. The in-well collective magnetic excitations are accounted for by setting $B_{\text {obs }}$ equal to the average hyperfine field calculated by Boltzmann statistics. ${ }^{49,50}$ For the present case, as $\left|K_{1}\right| \gg K_{\mathrm{Bu}}$, it is more adequate to use two-dimensional (2D) Boltzmann statistics rather than $3 \mathrm{D}$ Boltzmann statistics. The reduction of $B_{\mathrm{obs}}$ due to collective magnetic excitations can then be found from

$$
\begin{aligned}
B_{\mathrm{obs}} & =B_{0} \frac{\int_{0}^{\pi / 2} \exp \left(-K_{\mathrm{Bu}} V \sin ^{2} \phi / k T\right) \cos \phi d \phi}{\int_{0}^{\pi / 2} \exp \left(-K_{\mathrm{Bu}} V \sin ^{2} \phi / k T\right) d \phi} \\
& \approx B_{0}\left[1-\frac{k T}{4 K_{\mathrm{Bu}} V}\right]
\end{aligned}
$$

where the last expression is the low-temperature expansion for $k T \lesssim 0.1 \cdot K_{\mathrm{Bu}} V$. The variation of $B_{0}$ with temperature was assumed to follow that of bulk hematite after subtraction of $0.8 \mathrm{~T}$ below $T_{M}$ because of the absence of the Morin transition in the nanoparticles. ${ }^{1}$ We write the resulting Mössbauer spectrum as 


$$
G(\nu)=\int_{0}^{\infty} g\left(\nu, y \cdot E_{\mathrm{bm}}\right) f(y) d y,
$$

where $\nu$ is the velocity, $g\left(\nu, E_{b}\right)$ is the Mössbauer spectrum for a single energy barrier, $E_{b}=K_{\mathrm{Bu}} V$ and $f(y)$ is the volume weighted distribution of reduced energy barriers. The full expression for $g\left(\nu, E_{b}\right)$ can be found in Appendix B of Ref. 47. The other input parameters, which are not explicitly given in Eq. (23), are the magnetic hyperfine field, the relaxation time, the quadrupole interaction strength, the isomer shift and the intrinsic widths $W_{\mathrm{ij}}$ of lines $i$ and $j=7-i$. To avoid unrealistic sets of parameters the line widths were constrained to $W_{16} \geqslant W_{25} \geqslant W_{34}$ in the fitting. The values of $W_{\mathrm{ij}}$ and the quadrupole interaction strength were constrained to be identical for all spectra in a temperature series and the change of the isomer shift with temperature due to the second order Doppler shift was described using the Debye approximation. ${ }^{51}$ The full integrals in Eq. (22) were used to calculate $B_{\text {obs }}$ during the fitting.

The lines in Figs. 4 and 8 are the best fits of the spectra of samples $C 1$ and $C 2$ to the modified Blume-Tjon relaxation model, Eq. (23), obtained by simultaneous least-squares fits to the full temperature series of each of the samples. As can be seen from the figures all essential features of the spectra are well reproduced by the model. There are, however, some minor discrepancies at intermediate temperatures, which probably are due to small deviations of the actual barrier distribution from the log-normal distribution, and at high temperatures, which may be due to the failure of the simple Néel-Brown expression [Eq. (2)] for the relaxation time for $k T \approx E_{\mathrm{bm}}$ and the assumption of only two possible directions of the magnetic hyperfine field. The doublet, which is due to particles that exhibit fast superparamagnetic relaxation, is asymmetric at all temperatures, i.e., the line at negative velocity is broader than the line at positive velocity. This is clearly seen in the spectra of sample $C 2$ obtained at 375 and $495 \mathrm{~K}$ in a small velocity range (Fig. 8). This asymmetry is a result of relaxation effects for relaxation times of the order of $10^{-10} \mathrm{~s} .^{47,52}$ For shorter relaxation times the asymmetry is reduced. This explains why the doublet spectrum of sample $C 2$ is more symmetric at $495 \mathrm{~K}$ than at lower temperatures. The best fit to the temperature dependence of the secondorder Doppler shift was obtained using a Debye temperature $\Theta_{D} \sim 500 \mathrm{~K}$, which is similar to $\Theta_{D} \sim 600-700 \mathrm{~K}$ estimated from specific heat measurements on bulk hematite. ${ }^{1}$ Variations of $\pm 200 \mathrm{~K}$ on the values of $\Theta_{D}$ gave only a slight change of the quality of the fits.

For sample $C 1$ the isomer shift, extrapolated to zero temperature, was $\delta_{0}=0.49 \pm 0.01 \mathrm{~mm} \mathrm{~s}^{-1}$. The quadrupole interaction strength was $\varepsilon^{\prime} \equiv e Q V_{z z} / 4=0.21 \pm 0.01 \mathrm{~mm} \mathrm{~s}^{-1}$ corresponding to the quadrupole splitting $\Delta E_{Q}=2 \varepsilon^{\prime}=0.42$ $\pm 0.02 \mathrm{~mm} \mathrm{~s}^{-1}$ of the doublet at high temperatures and the quadrupole shift $\varepsilon=\varepsilon^{\prime} / 2 \cdot\left(3 \cos ^{2}(\pi / 2)-1\right)=-0.105$ $\pm 0.005 \mathrm{~mm} \mathrm{~s}^{-1}$ of the sextet at low temperatures. These parameters are in agreement with those expected for bulk hematite at low temperature in the absence of the Morin transition. The other parameters obtained from the fits of the spectra of the $C 1$ sample are $\tau_{0}=6.5_{-4.5}^{+10.5} \times 10^{-11} \mathrm{~s}, E_{\mathrm{bm}} / k$ $=590_{-120}^{+150} \mathrm{~K}, \quad \sigma=0.6 \pm 0.1$ and $B_{0}(T=0 \mathrm{~K})=53.3 \pm 0.2 \mathrm{~T}$. The uncertainties were estimated in the same manner as for the magnetization measurements. The larger value of $\tau_{0}$ corresponds to the lower value of $E_{\mathrm{bm}}$ and the larger value of $\sigma$.

It should be noted that the value of $E_{\mathrm{bm}}$ estimated by this method, is influenced by both collective magnetic excitations, which are related to the shape of the energy minimum, ${ }^{41}$ and superparamagnetic relaxation, which is related to the height of the energy barrier. If the shape of the energy barrier deviates from that given by Eq. (18) the change of $B_{\text {obs }}$ with temperature may therefore differ from Eq. (22). We checked this by estimating a value of $E_{\mathrm{bm}}$ from the low-temperature spectra obtained for $T<50 \mathrm{~K}$ where no doublet is present. The value of $E_{\mathrm{bm}}$ obtained in this way should mainly be determined from the collective magnetic excitations. In the fitting, $\tau_{0}$ and $\sigma$ were fixed to the values given above. The result was $E_{\mathrm{bm}}^{\mathrm{CME}} / k=620 \pm 50 \mathrm{~K}$. These spectra were also fitted using a distribution of hyperfine fields and the variation of the median field of the distribution (corresponding to a particle with energy barrier $E_{\mathrm{bm}}$ ) was fitted to Eq. (22) resulting in $E_{\mathrm{bm}}^{\mathrm{CME}} / k=640 \pm 50 \mathrm{~K}$. The values of $E_{\mathrm{bm}}^{\mathrm{CME}}$ do not differ significantly from the values of $E_{\mathrm{bm}}$ estimated from the full-temperature scan and hence Eq. (18) appears to be an adequate description of the anisotropy energy in the basal plane.

The parameters resulting from the analysis of the temperature series of Mössbauer spectra of the $C 2$ sample are $\delta_{0}=0.49 \pm 0.01 \mathrm{~mm} \mathrm{~s}^{-1}, \quad \varepsilon^{\prime}=0.24 \pm 0.01 \mathrm{~mm} \mathrm{~s}^{-1}, \quad \tau_{0}$ $=4.9_{-3.6}^{+5.3} \times 10^{-11} \mathrm{~s}, \quad E_{\mathrm{bm}} / k=470_{-90}^{+150} \mathrm{~K}, \quad \sigma=0.7 \pm 0.1 \quad$ and $B_{0}(T=0 \mathrm{~K})=53.0 \pm 0.2 \mathrm{~T}$. The obtained value of $\sigma$ is in good agreement with the value $0.7 \pm 0.3$ found from the TEM micrographs. The value of $\varepsilon^{\prime}$ for this temperature series is slightly larger than that obtained for sample $C 1$. This is due to the fact that we have measured on sample $C 2$ at higher temperatures resulting in a relatively larger weight of the high-temperature spectra and that the quadrupole splitting of the doublet at high temperatures, $\Delta E_{Q}=0.54$ $\pm 0.02 \mathrm{~mm} \mathrm{~s}^{-1}, \quad$ is slightly larger than $2 \varepsilon^{\prime}=0.48$ $\pm 0.02 \mathrm{~mm} \mathrm{~s}^{-1}$ expected from the fit of the full temperature series. The value of $\varepsilon$ in the low temperature spectra is $\varepsilon=$ $-0.098 \pm 0.005 \mathrm{~mm} \mathrm{~s}^{-1}$. A possible explanation of this is an enhancement of $\varepsilon^{\prime}$ due to surface effects or defects such as $\mathrm{OH}$ groups and vacancies. The contributions to the electric field gradient from defects presumably have random directions relative to the direction of the hyperfine field and will therefore mainly give rise to line broadening in the magnetically split spectra due to the angular dependence of the quadrupole shift [see Eq. (17)], but above $T_{\mathrm{Bm}}$ they will affect the average quadrupole splitting.

\section{G. Estimates of $\tau_{0}$ and anisotropy constants}

The two values of $\tau_{0}$ estimated from the Mössbauer spectroscopy measurements on samples $C 1$ and $C 2$, are in good agreement. The resulting average value is $\tau_{0}$ $=(6 \pm 4) \times 10^{-11} \mathrm{~s}$. The value, $\tau_{0}=1.8_{-1.3}^{+3.2} \times 10^{-11} \mathrm{~s}$, estimated from the magnetization measurements, is somewhat lower than the average value estimated from the Mössbauer spectra. Due to the different nature of the relaxation processes observed by magnetization measurements and Mössbauer spectroscopy, the two values do not have to be identical. They are, however, in agreement when the uncertainties are taken into account. 
The values of $\tau_{0}$ and $E_{\mathrm{bm}}$ found by Mössbauer spectroscopy, are in agreement with the estimates $\tau_{0} \sim 7 \times 10^{-12} \mathrm{~s}$ (the uncertainty was about an order of magnitude) and $E_{\mathrm{bm}} / k=500 \pm 200 \mathrm{~K}$ obtained from the broadening of the quasielastic component in inelastic neutron scattering spectra of the as-prepared sample assuming a single particle size. ${ }^{18}$ Mössbauer studies of this sample showed that it has a superparamagnetic blocking temperature similar to that of sample $C 1$ and the relaxation of the hematite particles in this sample is therefore not significantly affected by the presence of the impurity phase.

Due to the complexity of the relaxation process in the magnetization measurements it is difficult to relate $E_{\mathrm{bm}}^{\mathrm{magn}}$ directly to any of the barriers $K_{D} V$ and $K_{\mathrm{Bu}} V$. Using the bulk value, $K_{D}=2.0 \times 10^{3} \mathrm{Jm}^{-3}$ and the particle diameter of 16 $\pm 3 \mathrm{~nm}$ we calculate $K_{D} V / k=310_{-100}^{+200} \mathrm{~K}$ for sample $C 1$ (this value will be slightly larger than a value based on the median particle size but the effect on the obtained value is within the stated uncertainty). The value of $K_{D} V / k$ is significantly lower than $K_{\mathrm{Bu}} V / k$ but in good agreement with $E_{\mathrm{bm}}^{\mathrm{magn}} / k$ $=305 \pm 20 \mathrm{~K}$ and it thus supports the interpretation in terms of two different relaxation modes in the form of an in-plane and an out-of-plane fluctuation of $\mathbf{m}$.

\section{CONCLUSIONS}

The present work has shown that large quantities of pure $16 \mathrm{~nm}$ hematite particles can be prepared by a simple method. The small ferrihydrite component observed in the Mössbauer spectra of the as-prepared sample was removed with an oxalate treatment, and interparticle magnetic interactions were limited by coating the particles with oleic acid. No clear indication of an uncompensated moment was observed and an upper limit of about $350 \mu_{B}$ for this contribution was estimated. The magnetization of the particles was found to be slightly larger than the bulk value. This difference may be due to an uncompensated magnetic moment, a small amount $(\leqslant 2 \%)$ of ferrihydrite or a slight increase of the canting angle in the particles. Above the superparamagnetic blocking temperature, the Mössbauer spectra were found to consist of an asymmetric doublet for which the asymmetry decreases with increasing temperature. This is due to a superparamagnetic relaxation time of the order of
$10^{-10} \mathrm{~s}$ with the fluctuating hyperfine field perpendicular to the principal axis of the electric field gradient and is in accordance with the Blume-Tjon model for relaxation. By taking into account the particle size distribution and the effect of collective magnetic excitations we were able to simultaneously fit Mössbauer spectra obtained at temperatures ranging from $15 \mathrm{~K}$ to well above the superparamagnetic blocking temperature. This allowed us to determine the Néel relaxation pre-factor $\tau_{0}=(6 \pm 4) \times 10^{-11} \mathrm{~s}$ and the median energy barrier for relaxation in the basal plane $E_{\mathrm{bm}} / k=590_{-120}^{+150} \mathrm{~K}$ for sample $C 1$. These values are in accordance with those obtained from inelastic neutron-scattering studies. Simultaneous fits of the magnetization curves with a model for relaxation in three dimensions gave a slightly lower value of the pre-exponential factor, $\tau_{0}=1.8_{-1.3}^{+3.2} \times 10^{-11} \mathrm{~s}$, and an effective median energy barrier $E_{\mathrm{bm}}^{\mathrm{magn}} / k=305 \pm 20 \mathrm{~K}$, which corresponds well to the energy barrier $K_{D} V / k \approx 310 \mathrm{~K}$ estimated using bulk parameters. The median energy barrier, obtained from the magnetization measurements, is significantly lower than that obtained from the fit of the Mössbauer spectra. This difference is due to the presence of two different modes of magnetic relaxation, which are characteristic for the weakly ferromagnetic phase. One mode involves a 2D type of relaxation of the sublattice magnetization in the basal plane, while the other mode involves fluctuations of the weakly ferromagnetic moment out of the plane. The first effect gives rise to superparamagnetic behavior in both Mössbauer spectroscopy and magnetization measurements, while the second mainly affects the magnetization measurements. We therefore observe a $2 \mathrm{D}$ type of relaxation by Mössbauer spectroscopy, while the magnetization measurements are affected by a more 3D-like type of relaxation due to the effect of both in-plane and out-of-plane relaxation. The exponential pre-factor in the Néel law for the relaxation time may be different for these two modes.

\section{ACKNOWLEDGMENTS}

This work was supported by the Danish Council for Natural Sciences and the Danish Council for Technical Research. The authors would like to thank Peter Svedlindh and coworkers at Uppsala University, Sweden, for use of their equipment for magnetization measurements.
${ }^{1}$ A. H. Morrish, Canted Antiferromagnetism: Hematite (World Scientific, Singapore, 1994).

${ }^{2}$ R. M. Cornell and U. Schwertmann, The Iron Oxides, Structure, Properties, Occurrences and Uses (VCH, Weinheim, 1996).

${ }^{3}$ T. Nakamura, T. Shinjo, Y. Endoh, N. Yamamoto, M. Shiga, and Y. Nakamura, Phys. Lett. 12, 178 (1964).

${ }^{4}$ W. Kündig, H. Bömmel, G. Constabaris, and R. H. Lindquist, Phys. Rev. 142, 327 (1966).

${ }^{5}$ W. Kündig, K. J. Ando, R. H. Lindquist, and G. Constabaris, Czech. J. Phys., Sect. B 17, 467 (1967).

${ }^{6}$ A. M. van der Kraan, Phys. Status Solidi A 18, 215 (1973).

${ }^{7}$ D. Schroeer and R. C. Nininger, Jr., Phys. Rev. Lett. 19, 632 (1967).

${ }^{8}$ N. Yamamoto, J. Phys. Soc. Jpn. 24, 23 (1968).
${ }^{9}$ R. C. Nininger, Jr. and D. Schroeer, J. Phys. Chem. Solids 39, 137 (1978).

${ }^{10}$ G. J. Muench, S. Arajs, and E. Matijevic, Phys. Status Solidi A 92, 187 (1985).

${ }^{11}$ N. Amin and S. Arajs, Phys. Rev. B 35, 4810 (1987).

${ }^{12}$ G. J. Muench, S. Arajs, and E. Matijevic, J. Appl. Phys. 52, 2493 (1981).

${ }^{13}$ D. G. Rancourt, S. R. Julian, and J. M. Daniels, J. Magn. Magn. Mater. 49, 305 (1985).

${ }^{14}$ J. L. Dormann, J. R. Cui, and C. Stella, J. Appl. Phys. 57, 4283 (1985).

${ }^{15}$ P. Ayyub, M. Multani, M. Barma, V. R. Palkar, and R. Vijayaraghavan, J. Phys. C 21, 2229 (1988).

${ }^{16}$ R. V. Morris, D. G. Agresti, H. V. Lauer, Jr., J. A. Newcomb, T. 
D. Shelfer, and A. V. Murali, J. Geophys. Res. 94, 2760 (1989).

${ }^{17}$ R. Zysler, D. Fiorani, J. L. Dormann, and A. M. Testa, J. Magn. Magn. Mater. 133, 71 (1994).

${ }^{18}$ M. F. Hansen, F. Bødker, S. Mørup, K. Lefmann, K. N. Clausen, and P.-A. Lindgård, Phys. Rev. Lett. 79, 4910 (1997).

${ }^{19}$ R. D. Zysler, C. P. Arciprete, and M. I. Dimitrijewits, in NonCrystalline and Nanoscale Materials, Proceedings of the Fifth International Workshop on Non-Crystalline Solids, edited by R. Rivas and M. A. López-Quintela (World Scientific, Singapore, 1998), p. 481.

${ }^{20}$ L. Néel, in Low Temperature Physics, edited by C. DeWitt, B. Dreyfus, and P. G. DeGennes (Gordon and Beach, London, 1962), p. 411.

${ }^{21}$ J. T. Richardson, D. I. Yiagas, B. Turk, K. Forster, and M. V. Twigg, J. Appl. Phys. 70, 6977 (1991).

${ }^{22}$ S. A. Makhlouf, F. T. Parker, F. E. Spada, and A. E. Berkowitz, J. Appl. Phys. 81, 5561 (1997).

${ }^{23}$ L. Néel, Ann. Geophys. 5, 99 (1949).

${ }^{24}$ W. F. Brown, Jr., Phys. Rev. 130, 1677 (1963).

${ }^{25}$ M. C. Hobson, Jr. and H. M. Gager, J. Catal. 16, 254 (1970).

${ }^{26}$ J. A. Amelse, K. B. Arcuri, J. M. Butt, R. J. Matyl, L. H. Schwartz, and A. Shapiro, J. Phys. Chem. 85, 708 (1981).

${ }^{27}$ B. R. Morrison, A. H. Morrish, and G. T. Troup, Phys. Status, Solidi B 56, 183 (1973).

${ }^{28}$ P. J. Flanders and J. P. Remeika, Philos. Mag. 11, 1271 (1965).

${ }^{29}$ E. Kren, P. Szabo, and G. Konczos, Phys. Lett. 19, 306 (1965).

${ }^{30}$ U. Schwertmann, Z. Pflanzenernaehr., Dueng., Bodenkd. 105, 194 (1964).

${ }^{31}$ M. F. Hansen, C. Bender Koch, and S. Mørup (unpublished).

${ }^{32}$ K. J. Davies, S. Wells, and S. W. Charles, J. Magn. Magn. Mater. 122, 24 (1993).

${ }^{33}$ H. P. Klug and L. E. Alexander, X-ray Diffraction Procedures, (Wiley, New York, 1970), p. 514.

${ }^{34}$ K. O'Grady and A. Bradbury, J. Magn. Magn. Mater. 39, 91 (1983)
${ }^{35}$ E. Murad and U. Schwertmann, Am. Mineral. 6, 1044 (1980).

${ }^{36}$ E. Murad, in Iron in Soils and Clay Minerals, edited by J. W. Stucki, B. A. Goodman, and U. Schwertmann (Reidel, Dordrecht, 1988), p. 309.

${ }^{37}$ S. Mørup, J. A. Dumesic, and H. Topsøe, in Applications of Mössbauer Spectroscopy, edited by R. L. Cohen (Academic, New York, 1980), Vol. II, p. 1.

${ }^{38}$ F. van der Woude, Phys. Status Solidi 17, 417 (1966).

${ }^{39}$ J. M. D. Coey and P. W. Readman, Nature (London) 246, 476 (1973).

${ }^{40}$ K. Moorjani and J. M. D. Coey, Magnetic Glasses (Elsevier, Amsterdam, 1984), p. 169.

${ }^{41}$ S. Mørup, J. Magn. Magn. Mater. 37, 39 (1983).

${ }^{42}$ S. Mørup, P. H. Christensen, and B. S. Clausen, J. Magn. Magn. Mater. 68, 160 (1987).

${ }^{43}$ L. Tolber, W. Kündig, and I. Savic, Hyperfine Interact. 10, 1017 (1981).

${ }^{44}$ Q. A. Pankhurst and R. J. Pollard, in Mössbauer Spectroscopy Applied to Magnetism and Materials Science, edited by G. J. Long and F. Grandjean (Plenum, New York, 1993), Vol. 1, p. 77.

${ }^{45}$ E. P. Wohlfarth, Phys. Lett. A 70, 489 (1979).

${ }^{46}$ J. I. Gittleman, B. Abeles, and S. Bozowski, Phys. Rev. B 9, 3891 (1974).

${ }^{47}$ M. Blume and J. A. Tjon, Phys. Rev. 165, 446 (1968).

${ }^{48}$ J. L. García-Palacios and F. J. Lázaro, Phys. Rev. B 58, 14937 (1998).

${ }^{49}$ S. Mørup and H. Topsøe, Appl. Phys. 11, 63 (1976).

${ }^{50}$ S. Mørup, H. Topsøe, and J. Lipka, J. Phys. Colloq. 37, C6-287 (1976).

${ }^{51}$ See, for example, V. G. Bhide, Mössbauer Effect and its Applications (Tata McGraw-Hill, New Delhi, 1973), p. 93.

${ }^{52}$ A. M. Afanas'ev and V. D. Gorobchenko, Zh. Eksp. Teor. Fiz 66, 1406 (1974) [Sov. Phys. JETP 39, 690 (1974)]. 